\title{
Nutraceuticals in Chronic Coronary Syndromes: Preclinical Data and Translational Experiences
}

\author{
Chiara Tognola $a^{1,2} \cdot$ Maloberti Alessandro, $^{1,2}$ - Martina Milani ${ }^{1,2} \cdot$ Iside Cartella $^{1,2} \cdot$ Giovanni Tavecchia $^{1,2} \cdot$ \\ Enzo Grasso ${ }^{1,2} \cdot$ Jinwey Sun ${ }^{1,2} \cdot$ Cristina Giannattasio ${ }^{1,2}$
}

Received: 17 July 2020 / Accepted: 10 October 2020 / Published online: 30 October 2020

(c) The Author(s) 2020

\begin{abstract}
Non-pharmacological treatments have always been considered important in the management of Chronic Coronary Syndromes. Nutraceuticals ("Nutrition" + "Pharmaceutical") could fall both under the definition of non-pharmacological treatment and pharmacological one or, probably more correctly, in the middle of these two kinds of therapies. However, the word "nutraceuticals" never appears in the latest guidelines on this issue. This is probably determined by the fact that evidences on this topic are scarce and most of the published articles are based on preclinical data while translational experiences are available only for some molecules. In this review we will focus on nutraceutical strategies that act on the ischemic myocardium itself and not only on the cardiovascular risk factors. As demonstrated by the important number of papers published in recent years, this is an evolving topic and evaluated substances principally act on two mechanisms (cardiac energetics and ischemia-reperfusion damage) that will be also reviewed.
\end{abstract}

Keywords Nutraceutical $\cdot$ Chronic coronary syndromes $\cdot$ Ischemia-reperfusion injury $\cdot$ Cardiac energetics

\section{Introduction}

Non-pharmacological treatments have always been considered important in the management of chronic coronary syndromes (CCS - formerly called Chronic Ischemic Heart Disease). In fact, the last published guidelines on CCS spend a specific section on this topic [1]. Nutraceuticals ("Nutrition" + "Pharmaceutical") indicates a class of molecules/ supplements that are used in the prevention and/or treatment of disease [2]. They fall both under the definition of

This article is part of the topical collection on Nutraceuticals in Hypertension \& Cardiovascular Prevention.

Electronic supplementary material The online version of this article (doi:https://doi.org/10.1007/s40292-020-00416-8) contains supplementary material, which is available to authorized users.

Maloberti Alessandro

alessandro.maloberti@ospedaleniguarda.it

1 Cardiologia IV, Dipartimento A. De Gasperis, Ospedale Niguarda Ca' Granda, Piazza Ospedale Maggiore 3, 20159 Milan, Italy

2 School of Medicine and Surgery, Milano-Bicocca University, Milan, Italy non-pharmacological treatment and pharmacological one or, probably more correctly, in the middle of these two kinds of therapies. However, the word "nutraceuticals" never appears in the previously cited guidelines. This is probably determined by the fact that evidences on this topic are scarce and most of the published articles are based on preclinical data while translational experiences are available only for some molecules. In this review we will focus on nutraceutical strategies that act on the ischemic myocardium itself. As demonstrated by the important number of papers published in recent years, this is an evolving topic and evaluated substances principally act on two mechanisms (cardiac energetics and ischemia-reperfusion damage) that will be also reviewed.

Nutraceuticals in CCS could be also used to help controlling risk factors in patients on optimal medical therapies who don't reach specific cut-off or are intolerant to some drug classes. The discussion of this approach is beyond the purpose of this review but focused papers have been published in recent years [3, 4].

Table 1 summarized the effects on nutraceutical in CCS both at the preclinical and clinical levels. Furthermore Fig. 1 sum up the cardiac energetics pathways and point out where nutraceuticals acts. Similarly, Fig. 2 showed the principal pathways of ischemia-reperfusion damage and nutraceuticals effects. 


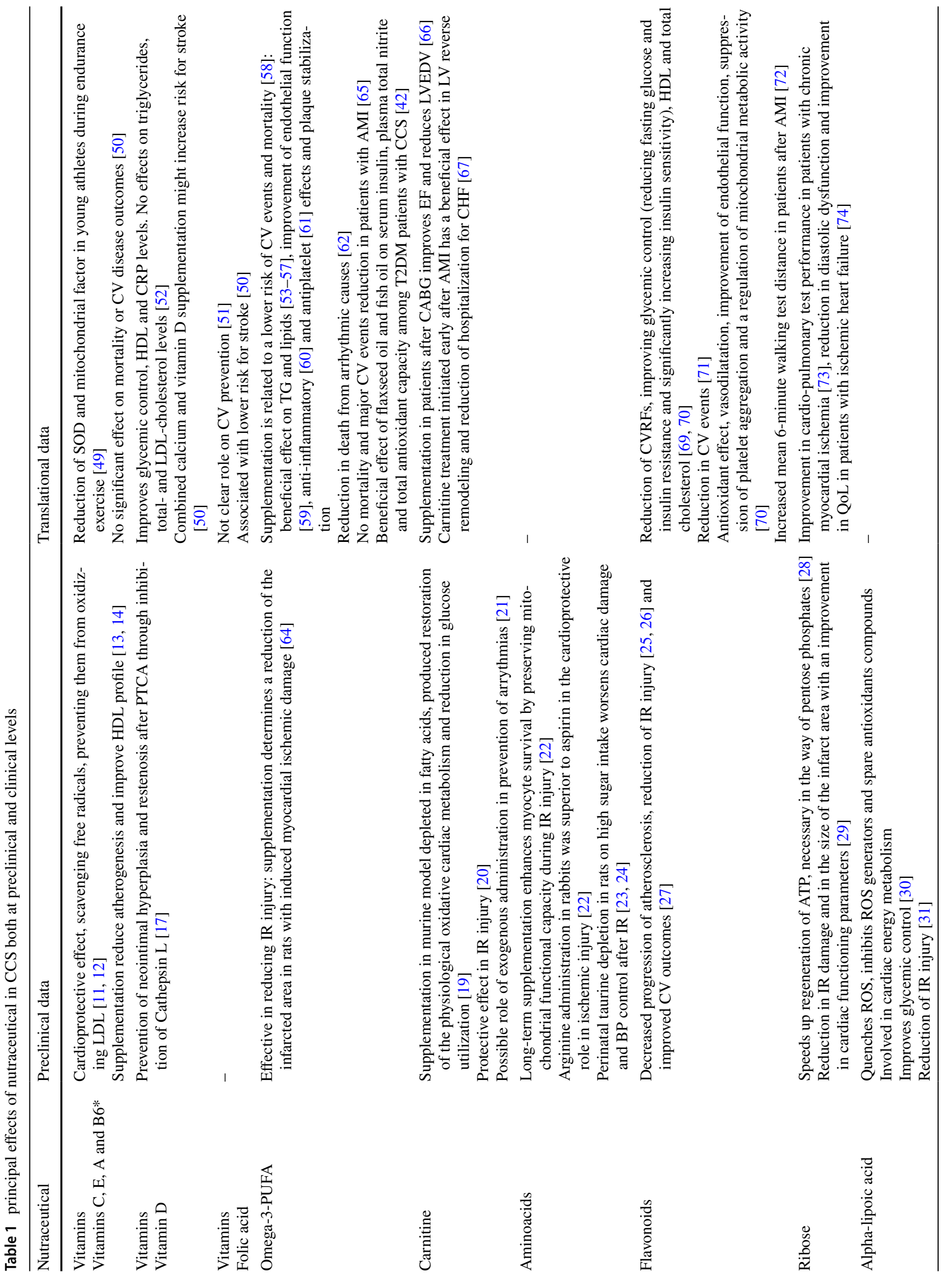




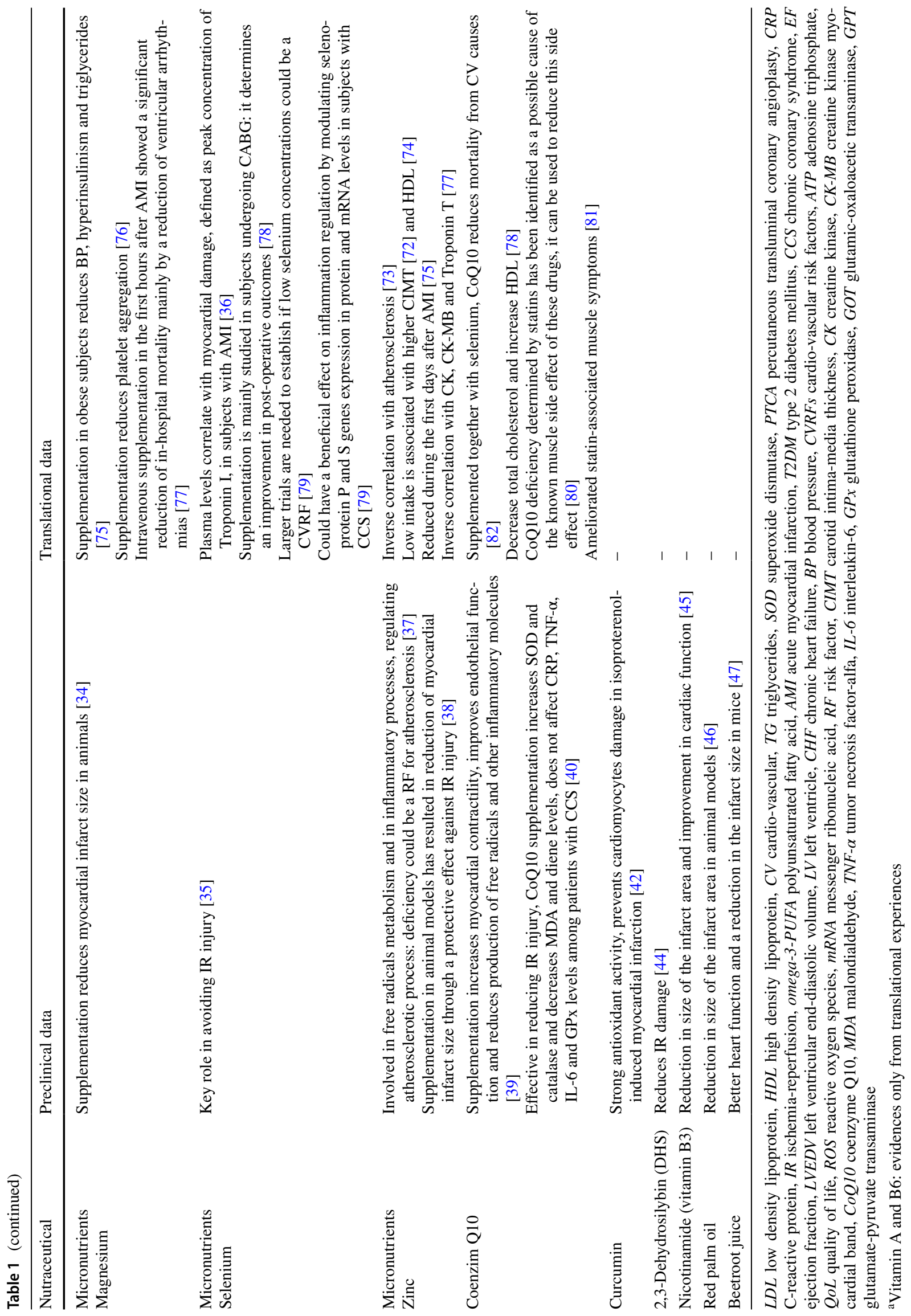




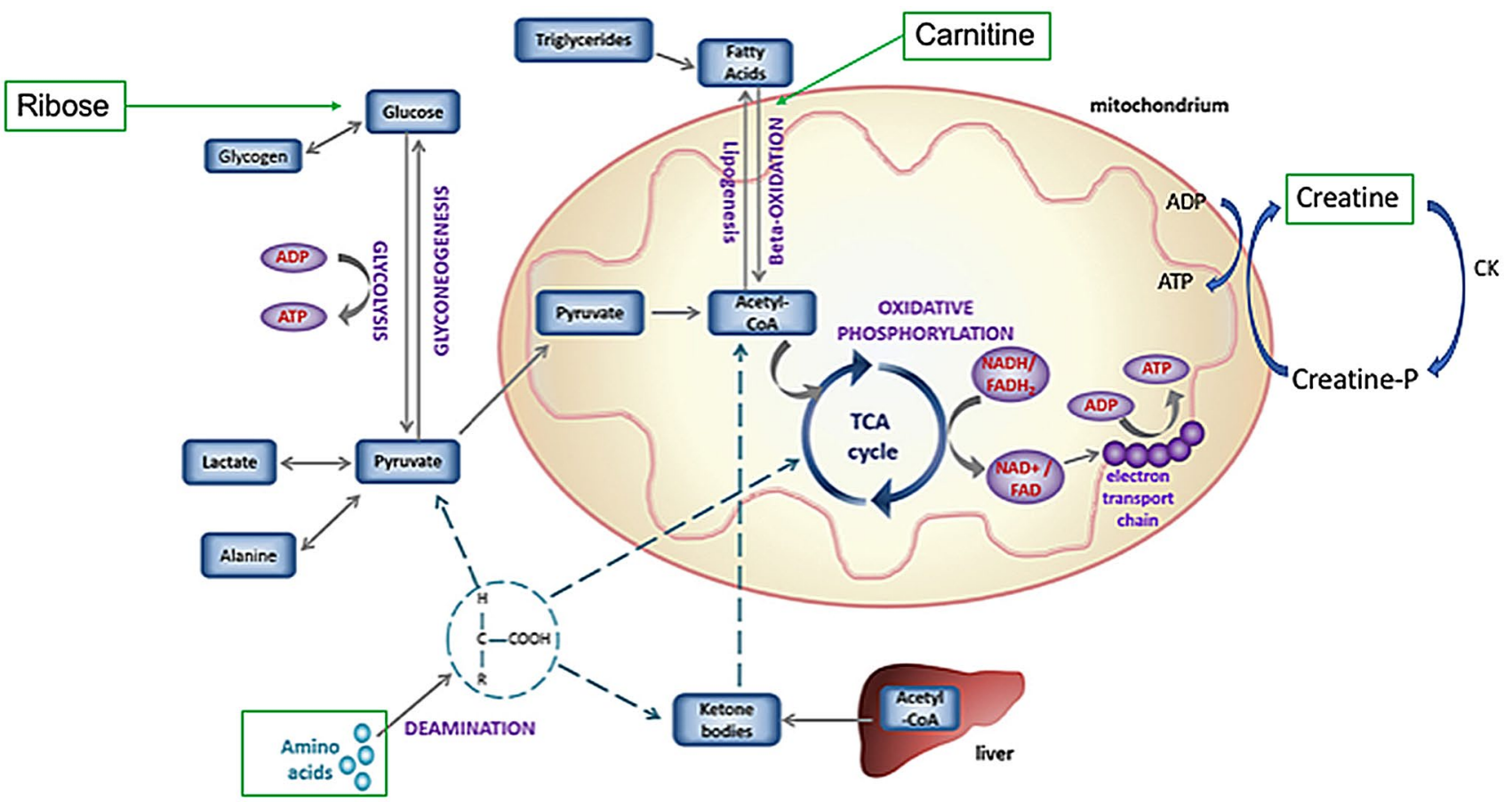

Fig. 1 Cardiac energetics pathways and effects of nutraceuticals. Modified with permission from Malandraki-Miller S, Lopez CA, AlSiddiqi H, Carr CA. Changing Metabolism in Differentiating Cardiac
Progenitor Cells-Can Stem Cells Become Metabolically Flexible Cardiomyocytes? Front Cardiovasc Med. 2018 Sep 19;5:119.

Fig. 2 Principal pathways of ischemia-reperfusion damage and nutraceuticals effects on it. Modified with permission from Xia Z, Li H, Irwin MG. Myocardial ischaemia reperfusion injury: the challenge of translating ischaemic and anaesthetic protection from animal models to humans. Br J Anaesth. 2016 Sep;117 Suppl 2:ii44-ii62.

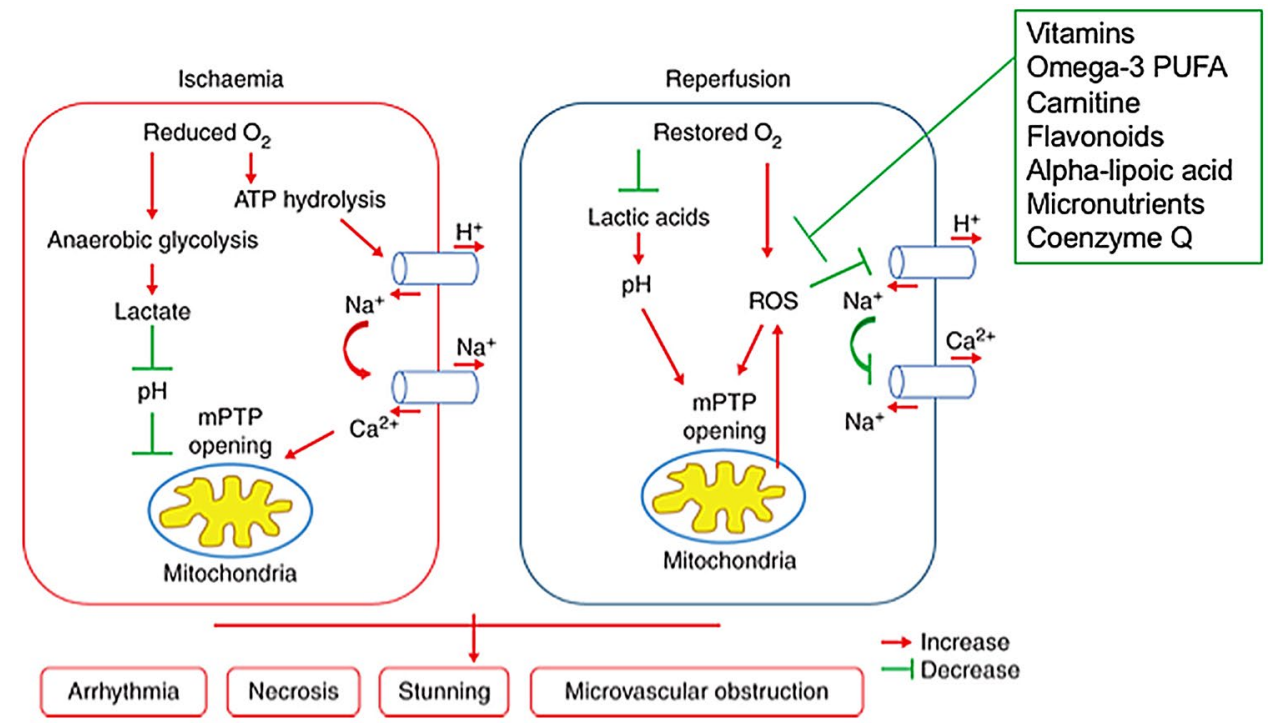

\section{Methods}

Relevant studies were identified by PubMed and Embase sources up to June 2020. Only English language were allowed. The first search was performed with the following keywords: (nutraceutical OR dietary supplement)
AND (chronic coronary syndrome OR ischemic heart OR heart energetics OR ischemia-reperfusion). After excluding duplicates, 224 title and abstract have been screened independently by two researchers (CT, AM). After the first screening, 136 relevant studies were retrieved in full text and 83 four studies were finally used to elaborate this revision. 


\section{Cardiac Energetics}

Heart work consumes a large amount of energy and its depletion is common in various cardiological diseases. Under normal conditions the heart draws $70 \%$ of the needed energy from the $\beta$-oxidation of fatty acids (or fatty-acids oxidation, FAO) while $10-30 \%$ is derived from the oxidation of glucose and a small part from ketones, lactates and aminoacids [5]. However, the pathways from which heart derives energy depend on the metabolic state and the availability of oxygen with the possibility to shift among them. The use of glucose, which is the main way of energy accumulation in the other cells of the organism, is a minor energetic source for the heart. Once transported inside the cell through its membrane conveyors it is transformed into pyruvate and then conveyed inside the mitochondria, where it will access the cycle of tricarboxylic acids for the formation of ATP. The amount of oxygen, as well as the ratio between nicotinamide adenine dinucleotide (NAD) and it's oxidate form (NADH) are very important regulators of this cycle. Especially under hypoxia conditions (as both the case of acute coronary syndromes-ACS - and CCS) pyruvate is moved preferably to lactate pathway with the formation of fewer energetic adenosine triphosphate (ATP) molecules.

As previously mentioned, the main heart energetic pathway is the FAO, both with circulating fatty acids and, when missing, reserves of triglycerides. After the addition of the acylCoa in the cytosol, the fatty acid is transported to the mitochondrion through a specific transport system that requires carnitine for its proper functioning. The dependent carnitine transport represents the bottleneck of the fatty acid oxidation system, since its failure to pass inside the mitochondrion does not allow the process to continue properly. Once inside the mitochondria, the process of $\beta$-oxidation and energy production begins: long-chain acyl-CoA metabolism in the mitochondrial matrix occurs via the $\beta$-oxidation pathway involving the activity of specific enzymes such as acyl-CoA dehydrogenase, enoyl-CoA hydratase and others. Each cycle of FAO results in the production of acetyl-CoA, FADH2, and NADH, which can also modulate the activity of the above enzymes through an inhibitory feedback. An acute increase in workload of healthy hearts, for instance during exercise or $\beta$-adrenergic stimulation, increases myocardial FAO [6].

FAO is less energy efficient than glucose oxidation, theoretically requiring $11-12 \%$ more oxygen for a given amount of ATP produced [7].

\section{Ischemia-Reperfusion Injury}

Many research studies have focused on the role of various nutraceutical molecules on reperfusion injury, namely the injury induced by the restoration of blood flow and oxygen in the area that was previously ischemic. The coronary revascularization and the restoration of a correct oxygen supply to the ischemic tissue determine a series of molecular pathways that, if not controlled, can determine further injury.

During ischemia the absence of oxygen in the cardiomyocyte causes a shift towards anaerobic metabolism, the production of lactates and therefore cellular acidosis. This determines the increase in intracellular sodium and calcium with rupture of the mitochondria and, consequently, cell death [8]. NAD/NADH ratio plays a central role in regulating cardiomyocyte metabolism. It is also able to determine post-transcriptional regulations and to adjust the activity of the enzymes involved in the response to DNA damage and the signaling of intracellular calcium. In fact, as we will see, the supplementation of its derivatives plays an important role in the search for a possible therapy for reperfusion injury.

During the reperfusion period, the recovery of oxygen determines the formation of an important amount of reactive oxygen species (ROS) that could contribute to cell death. Strategies that try to reduce this reperfusion injury could both act conditioning the heart to ischemia (pre-conditioning, in order to reduce the injury when it will occur) or regulating the process acting on nitric oxide and ROS formation [9].

\section{Preclinical Data}

\subsection{Vitamin Supplements}

\subsubsection{Vitamin C and E}

ROS levels have been associated with an increased risk of stroke and myocardial infarction [10,11]. Both vitamin C and vitamin $\mathrm{E}$ have shown a cardioprotective effect, the former scavenging free radicals, the latter neutralizing them through peroxidation, preventing them from oxidizing low density lipoprotein (LDL) [12, 13].

However, data in favor of their supplementation are poor and come from animal models: there is evidence of less atherogenesis and protective remodeling of high density lipoprotein (HDL) profile [14].

\subsubsection{Vitamin D}

Vitamin D could have a role in preventing neointimal hyperplasia (NIH) and restenosis after percutaneous transluminal coronary angioplasty (PTCA). A preclinical study evaluated the modulatory effect of vitamin D in coronary arteries of atherosclerotic swine, showing that vitamin D could inhibit Cathepsin L (CTSL), an endosomal and lysosomal protease with elastase and collagenase activity, associated with 
vascular remodeling. In fact, increased expression of CTSL correlates with the formation of NIH in the PTCA-injured coronary arteries and, in the presence of sufficient or supplemented levels of vitamin D in blood, CTSL expression is significantly reduced. In addition, the greatest restenosisassessed with Optical Coherence Tomography performed after PTCA—happens in vitamin D deficient group [15].

\subsection{Omega-3-PUFA}

The major evidence about omega-3-polyunsaturated fatty acids (PUFA) in preclinical experience, is the demonstration that they are effective in reducing IR injury: their supplementation determines a reduction of the infarcted area in rats with induced myocardial ischemic damage [16].

\subsection{Carnitine}

Carnitine is essential for the transfer of long-chain fatty acids across the inner mitochondrial membrane for subsequent $\beta$-oxidation. Fatty acids are the substrate for myocardial energy production, and in many cardiac diseases a depletion in carnitine has been demonstrated [17].

The role of exogenous carnitine supplementation in the improvement of cardiac metabolism is under investigation. In preclinical studies, in the murine model depleted in fatty acids, carnitine supplementation produced restoration of the physiological oxidative cardiac metabolism and reduction in glucose utilization [18].

Furthermore, effects of carnitine on post-ischemic myocardial metabolism has been studied in isolated rat heart. Carnitine was shown to have a protective effect in ischemiareperfusion injury. With oxygen restoration in myocytes, a large amount of acetyl-CoA gets bound to fatty acids chains and accumulates in the cytosol, causing oxidative stress. Exogenous supplementation of carnitine improves transport of fatty acids inside mitochondria and conversion of ATP production into cardiac work [19] triggering cardioprotective effects through reduced oxidative stress, inflammation and necrosis of myocytes.

Additionally, improvement in cardiac metabolism and ventricular function suggest a possible role of exogenous administration in prevention of arrhythmias. However, just a few studies have been conducted so far in animal models demonstrating a reduction in arrhythmic events and further studies are needed to investigate this aspect.

\subsection{Aminoacids}

In preclinical studies, long-term supplementation with mixed aminoacids enhanced myocyte survival by preserving mitochondrial functional capacity during ischemic-reperfusion injury. Arginine administration in rabbits was superior to aspirin in the cardioprotective role in ischemic injury [20].

Furthermore, it has been shown that perinatal taurine depletion in rats worsens cardiac damage and arterial pressure control after ischemia/reperfusion (IR) [21]. These adverse effects are exacerbated by high sugar intake from weaning onward. Taurine supplementation could ameliorate IR damage through inhibition of both cardiac and systemic renin-angiotensin system overactivity.

This is an interesting topic because, nowadays, an increasing number of women follow a vegetarian or vegan diet: this results in their fetuses being depleted of taurine, as this compound can be found only in animal derivative products. Furthermore, children consume high levels of sugar, due in part to the excessive amounts of sugar in processed foods. This combination could place the child at risk for adverse cardiovascular effects later in life. More data are needed to understand if taurine supplementation has a beneficial effect in IR damage also in cases without previous taurine depletion.

\subsection{Flavonoids}

Flavonoids are a subgroup of polyphenols, namely a class of molecules characterized by numerous phenol groups. We can find them in most plants: in fact, more than 5000 plants have been classified with a high content, like onions, broccoli, apples, blueberries, tea and especially grapes, whose skin contains resveratrol in abundance.

Several studies have reported that phloroglucinol has antioxidant, anti-inflammatory and antihyperglycemic properties [22]. A recent study demonstrated that treatment with phloroglucinol prevents cardiovascular damage in diabetic rat hearts: the treatment was able to protect the heart after IR by preventing haemodynamic changes, preserving antioxidant enzymes, and reversing biochemical and histopathological changes [23].

In addition, there are many studies that have tested its preclinical efficacy in animal models on decreased progression of atherosclerosis, on the reduction of IR injury and thus on improved cardiovascular outcomes (reduced premature death and attenuated myocardial infarction and disfunction) $[24,25]$.

\subsection{Ribose}

Ribose plays an important role in cardiac energy. In conditions of ischemia, in fact, the regeneration of ATP is speeded-up by the presence of ribose as necessary in the way of pentose phosphates [26]. At a pre-clinical level, its supplementation has shown a reduction in IR damage and in the size of the infarct area with an improvement in the cardiac functioning parameters [27]. 


\subsection{Alpha-Lipoic Acid}

Lipoic acid takes part in several antioxidant processes since it is able to directly quench ROS, inhibit ROS generators and spare antioxidants compounds. It is also involved in cardiac energy metabolism being a cofactor of enzyme complexes that catalyze the oxidative decarboxylation of pyruvate and other ketoacids. Eventually, it plays an important role in glucose metabolism and can be used as a dietary supplement in diabetic subjects to improve glycemic control [28]. Even in this case, several preclinical evidences show lipoic acid effectiveness in reducing IR injury is available [29], while definitive evidence on humans is still missing.

\subsection{Micronutrients}

Among the components of this class, those with more marked importance for CCS are magnesium, selenium and zinc.

\subsubsection{Magnesium}

Regarding the first one, circulating levels of magnesium have been indirectly associated with cardiovascular events and with the prevalence of diabetes mellitus and hypertension [30]. Indeed, magnesium is the second most abundant intracellular electrolyte after potassium and has important functions as a cofactor of enzymatic reactions involved in carbohydrate metabolism but also in vascular tone and endothelial function [31]. Concerning CCS, preclinical evidence has shown that magnesium supplementation leads to a reduction of myocardial infarct size in animals [32].

\subsubsection{SeleniumSEC3}

On the other hand, selenium has a fundamental role for the functioning of enzymes involved in free radical cleaning systems, thus having a key role in avoiding IR injury [33]. Indeed, it has been shown that its plasma levels correlate with myocardial damage, defined as peak concentration of Troponin I, in subjects with myocardial infarction [34].

\subsubsection{Zinc}

Zinc is required for the catalytic activation of more than 300 enzymes, it is involved in the metabolism of free radicals and it plays an important role in inflammatory processes. Given its antioxidant and anti-inflammatory functions, zinc regulates atherosclerotic process and its deficiency could be a risk factor for atherosclerosis [35]. Furthermore, its supplementation in animal models has resulted in a reduction of myocardial infarct size through a protective effect against IR injury [36]. In fact, in a myocardial infarction dog model generated by left coronary artery branch occlusion, administration of zinc sulphate $(10 \mathrm{mg} / \mathrm{kg})$ hours before coronary occlusion significantly reduced the infarct size by almost half. In two Langendorff perfused rat heart studies, administrating zinc with zinc ionophore pyrithione during reperfusion and maintaining high levels of cytosolic zinc by postconditioning greatly improved myocardial recovery after IR to almost $100 \%$, and decreased arrhythmias and infarct sizes more than twofold [37, 38].

\subsection{Coenzyme Q10}

Coenzyme Q10, or ubiquinone, is an organic molecule contained in the mitochondria. It is used in fundamental reactions for cellular energy production. Its supplementation increases myocardial contractility, improves endothelial function and reduces the production of free radicals and others inflammatory molecules [39]. In fact, it was demonstrated that Coenzyme Q10 is effective in reducing IR injury in animal studies on experimental models of myocardial infarction [40]. Systemic inflammation and oxidative stress significantly contribute in developing CAD. In a meta-analysis CoQ10 supplementation was demonstrated to increase superoxide dismutase (SOD) and catalase and decrease malondialdehyde and diene levels, but did not affect C-reactive protein (CRP), tumor necrosis factor $\alpha$, interleukin-6, and glutathione peroxidase levels among patients with CCS.

\subsection{Others}

In the end, the following nutraceutical substances have been evaluated in animal models mainly on the basis of their ability to reduce IR damage:

- Curcumin is a nutraceutical compound, derived from Curcuma longa, with strong antioxidant activity but whose clinical use is limited due to its low bioavailability. However, nanotechnology has made possible the development of curcumin nanoparticles with increased permeability, absorption and resistance to metabolic processes. Animal models have demonstrated that curcumin nanoparticles exert greater antioxidant effects compared to conventional curcumin and that curcumin nanoparticles better prevent cardiomyocytes damage in isoproterenol-induced myocardial infarction [41].

- 2,3-Dehydrosilybin (DHS) a component of silymarin extract from the thistle seed [Silybum marianum]. This compound has antioxidant and cytoprotective activities and has been evaluated in isolated hearts of rats subjected to IR. Hearts treated with this product had a significantly lower post-reperfusion area of infarction [42]. 
- Nicotinamide (vitamin B3) supplementation is able to act on NAD + and in various animal models it has resulted in a reduction in the size of the infarct area and an improvement in cardiac function. It can be synthesized from tryptophan as nicotinic acid, nicotinamide and nicotinamide riboside.

- Red palm oil: thanks to its antioxidant properties, it has also shown a reduction in the size of the heart attack following IR in animal models [43].

- Beetroot juice: through the metabolic effect of the increase in cyclic GMP, beet juice derivatives have shown better heart function and a reduction in the size of the heart attack after ischemic damage induced in mice [44].

\section{Translational Experiences}

\subsection{Vitamin Supplements}

\subsubsection{Vitamin $C, E, A$ and $B 6$}

Multivitamins supplementation in humans has been assessed in young athletes during endurance exercise [45]. Although this did not determine variations in the most common indices for the evaluation of muscle oxidative stress, it was still possible to detect a reduction of some pathways, such as SOD and mitochondrial factor A. Considering that the myocardium has many similarities with striated muscle, lots of authors decided to start investigating through specific studies to extend this strategy to coronary artery disease. But a recent meta-analysis assessed the role of multivitamins supplementations and concluded that they have no significant effect on mortality or cardiovascular disease outcomes [46].

Finally, as a results of the previously cited meta-analysis, Vitamin A, Vitamin B6 and iron had no significant effect on mortality or cardiovascular events [47].

\subsubsection{Vitamin D}

Vitamin D, according to a recent meta-analysis, had the beneficial effects on improving glycemic control, HDLcholesterol and CRP levels among patients with cardiovascular disease, though it did not affect triglycerides, total- and LDL-cholesterol levels [48]. But, on the other hand another recent meta-analysis showed that combined calcium and vitamin D supplementation must be done carefully as it might increase risk for stroke [49].

\subsubsection{Folic Acid}

Folic acid appears to be implicated in cardiovascular events, as it reduces homocysteine levels. A meta-analysis published in 2006 failed in demonstrating a convincing effect of folic acid supplementation on reduction of cardiovascular events but, up to date, some trials on large numbers are underway to clarify the role of folic acid in cardiovascular prevention. In a recent meta-analysis folic acid was instead associated with lower risk for stroke [50].

\subsection{Omega-3-PUFA}

In addition to their well-known effect on triglycerides and lipids, many epidemiological studies and meta-analysis have shown that the supplementation of omega-3-PUFA is related to a lower risk of cardiovascular events [51]. More specifically, an Italian multicentric study (GISSI) has shown that supplementing $1 \mathrm{~g}$ of PUFA per day determines a reduction in mortality risk even only after 3 months of treatment [52]. Furthermore, a novel study by Raygan et al. revealed the beneficial effect of flaxseed oil and fish oil (two different sources of omega- 3 fatty acids) on serum insulin, plasma total nitrite and total antioxidant capacity among type 2 diabetic patients with CCS [41].

Several mechanisms have been proposed to explain the beneficial effect of omega-3: improvement of endothelial function, anti-inflammatory, and antiplatelet effects and plaque stabilization [53, 54]. Anyway, it seems that the most important effect on mortality was attributable to a reduction in death from arrhythmic causes. Recently, another study supported this hypothesis, showing that PUFA supplementation in patients with post-ischemic dilated cardiomyopathy carrying implantable cardiac defibrillator was associated with fewer episodes of malignant ventricular tachyarrhythmias.

In contrast with these evidences, a recently published meta-analysis has examined 10 randomized controlled trials in order to assess whether Omega-3 fatty acids affects mortality, morbidity and adverse events in patients with acute myocardial infarction. The authors, based on the results of the subgroup analysis, found out that Omega 3 fatty acids do not reduce mortality and major cardiovascular events in patients with acute myocardial infarction [55].

In 2019 were published the results of an important randomized controlled trial (REDUCE-IT, Reduction of Cardiovascular Events with Icosapent Ethyl-Intervention Trial) comparing incidence of first and subsequent cardiovascular events (death, nonfatal myocardial infarction, nonfatal stroke, coronary revascularization and hospitalization for unstable angina) in statin-treated patients with elevated triglycerides and cardiovascular disease or diabetes taking icosapent ethyl $4 \mathrm{~g} /$ day versus placebo. The study demonstrated that the administration of icosapent ethyl significantly reduces the burden of first, subsequent, and total ischemic events compared to placebo ( $25 \%$ relative risk reduction and a $4.8 \%$ absolute risk reduction, number needed to treat of 
21). 8179 patients were followed for about 5 years: those treated with icosapent ethyl had a lower incidence of all cardiovascular events (61 vs. 89 per 1000 patient-years for icosapent ethyl versus placebo, respectively; rate ratio: 0.70; 95\% confidence interval $0.62-0.78 ; p<0.0001$ ) [56].

A complete discussion on the effect of triglycerides on cardiovascular outcomes is beyond the scope of our paper and would required a focused paper. However, this argument has been recently discussed in many editorial comments [57, 58].

\subsection{Creatine}

Creatine supplementation during ischemia or as a pre-treatment has resulted in arrhythmias prevention and improvement of many parameters of cardiac function. Therefore, its administration as a pre-treatment in patients at high risk of cardiovascular events could be effective, even if definitive demonstrations from randomized controlled trials are required. Nowadays, creatine use in clinical practice is limited to cardioplegic solutions for explanted heart in view of the transplant.

\subsection{Carnitine}

In clinical studies, carnitine supplementation in patients who underwent coronary artery bypass grafting (CABG) determined an improvement in the ejection fraction and a reduction in end-diastolic volume of the left ventricle [17].

Carnitine treatment initiated early after acute myocardial infarction showed a beneficial effect in left ventricle reverse remodeling, with significant attenuation of ventricular dilatation and hospitalization for congestive heart failure in the first year after infarction.

Moreover, carnitine appears to possess considerable potentials for the long-term treatment of patients with heart failure attributable to dilated cardiomyopathy. In these patients, long term administration for a period of 3 years was correlated with increased survival and improvement in cardio-pulmonary exercise testing, both in peak oxygen consumption and carbon dioxide production [59].

\subsection{Flavonoids}

These molecules have been studied extensively for their antiischemic effect which is due to:

- Reduction of cardiovascular risk factors. An interesting randomized, double-blind, placebo-controlled trial showed that a 4-week supplementation of resveratrol in patients with type 2 diabetes mellitus and CCS had beneficial effects on metabolic status, improving glycemic control (reducing fasting glucose and insulin resistance and significantly increasing insulin sensitivity), HDLcholesterol levels and total-/HDL-cholesterol ratio [60].

- An antioxidant effect, vasodilatation, improvement of endothelial function, suppression of platelet aggregation and a regulation of mitochondrial metabolic activity [60].

Many epidemiological studies have correlated the consumption of flavonoids in specific populations to a reduction in cardiovascular events, as confirmed also by some reviews [61].

In an open-label randomized clinical trial patients affected by acute myocardial infarction the mean 6-min walk test distance increased significantly more in the bilberry group compared to the control group: mean difference 38 months at follow-up (95\% confidence interval 14-62, $p=0.003$ ) [62].

\subsection{Ribose}

At clinical level was demonstrated an improvement in cardio-pulmonary test performance in patients with CCS [63], a reduction in diastolic dysfunction and an improvement in the quality of life in patients with ischemic heart failure [64].

\subsection{Micronutrients}

\subsubsection{Magnesium}

Hence the theoretical basis that would associate the deficiency of magnesium with the presence of diabetes mellitus and hypertension but also with cardiovascular events, to date, a single small randomized double-blind placebocontrolled trial has evaluated magnesium supplementation in obese subjects by assessing its effect on cardiovascular risk factors. Individuals in the intervention group received $382 \mathrm{mg}$ of magnesium once daily during 4 months, witnessing a significant reduction in blood pressure, hyperinsulinism and triglycerides [65].

It was demonstrated that magnesium causes a reduction in platelet aggregation following magnesium supplementation [66]. In addition, some trials of intravenous magnesium supplementation in the first hours after an acute myocardial infarction have been performed, and subsequently metaanalyzed, identifying a significant reduction of in-hospital mortality mainly determined by a reduction of the incidence of ventricular arrhythmias and arrhythmias related deaths [67]. Nevertheless, it is also important to draw attention to the possible toxic effects of IV magnesium supplementation such as flushing, severe hypotension and bradyarrhythmia.

\subsubsection{Selenium}

The supplementation of selenium is now mainly studied in subjects undergoing CABG surgery, in which it determines 
an improvement in post-operative outcomes [68]. In fact, it was hypothesized that selenium supplementation had beneficial effect of inflammation regulation by modulating selenoprotein $\mathrm{P}$ and $\mathrm{S}$ genes expression in protein and $\mathrm{mRNA}$ levels in subjects with CCS [69]. With regard to primary prevention, selenium supplementation has led to heterogeneous results in various trials and meta-analysis. We need larger trials to establish if low selenium concentrations could be a cardiovascular risk factor.

\subsubsection{Zinc}

The association between zinc deficiency and the development of cardiovascular diseases has been supported by numerous studies. Using carotid intima-media thickness (IMT) as a valuable marker, the subclinical atherosclerosis was measured and explored for its relationship with the dietary zinc intake [70]. In the middle-aged and elderly populations, the low zinc intake group showed a higher IMT than the high zinc intake group. The inverse correlation of zinc intake and atherosclerosis risk was also reported in another cross-sectional study [71]. Lower consumption of dietary zinc was associated with low HDL cholesterol levels as well as an increased prevalence of coronary artery disease. A very recent meta-analysis of randomized primary prevention trials failed to show significant beneficial effects of the nutrient on cardiovascular disease [72].

An earlier study showed that patients with myocardial infarction showed a highly significant reduction in serum zinc within the first 3 days, compared to control or borderline groups [73]. In a meta-analysis with 2886 subjects from 41 case-control studies, the authors revealed that the subjects with MI had lower zinc levels in serum and hair than healthy controls [74]. Moreover, a recent study using zinc quartiles also demonstrated a significant inverse correlation between serum zinc levels and serum creatine kinase (CK), CK-MB, and cardiac troponin T levels [75]. This study further showed the prevalence rate of myocardial infarctions decreased with increasing zinc quartiles.

\subsection{Coenzyme Q10}

It was proved that the supplementation of Coenzyme Q10, together with Selenium, reduce the mortality from cardiovascular causes in a cohort of patients in Sweden [76]. A recent meta-analysis showed that taking Coenzyme Q10 significantly decreased total cholesterol and increased HDL cholesterol levels in patients with CAD without any effect on LDL-cholesterol, triglycerides and Lp(a) levels [77].

Statins, the milestone in lipid-lowering treatment, inhibit hydroxyl-methylglutaryl coenzyme A reductase, a rate limiting enzyme not only in cholesterol synthesis but also in the synthesis of farnesyl pyrophosphate that is essential for Coenzyme Q10 biosynthesis, thus explaining the link between statin use and Coenzyme Q10 deficiency [78]. In fact, a recent meta-analysis of 12 RCTs involving 1776 participants concluded that, compared to the placebo, statin treatment resulted in a reduction of circulating Coenzyme Q10 independently from statin solution, intensity, and treatment time [79]. No study has yet been designed to demonstrate that Coenzyme Q10 supplementation could prevent statin-related myalgia. However, a meta-analysis of 12 RCTs involving 575 patients concluded that, compared to the placebo, $\mathrm{CoQ}_{10}$ supplementation ameliorated statinassociated muscle symptoms, such as muscle pain, muscle weakness, muscle cramping and muscle tiredness, whereas no reduction in plasma creatine kinase levels was observed after Coenzyme Q10 supplementation [80]. These positive effects are usually achieved only with high dosages of Coenzyme Q10 ( $\geq 200 \mathrm{mg} /$ day).

Although there are no definitive demonstrations yet, it can be used to try to reduce this side effect in patients taking lipid-lowering drugs, as it was clinically proved that Coenzyme Q10 supplementation could be able to improve selfperceived fatigue in healthy subjects, in obese patients, and in patients affected by fibromyalgia [81].

\subsection{Crocetine}

A recent RCT demonstrated that the supplementation of crocetine in CCS patients alters the expression of atherogenic genes and endothelial cell adhesion molecules. It revealed that serum circulating homocystein, heart-type fatty acid binding protein, intercellular adhesion molecule 1 , vascular cell adhesion molecule 1 and monocyte chemoattractant protein 1 decreased significantly in the crocetin group, while high-density lipoprotein (HDL) significantly increased. Also, systolic and diastolic blood pressures decreased significantly in the crocetin group in comparison with the placebo. Concerning these results, it appears that crocetin could be considered as a promising anti-atherogenic candidate for future studies.

\section{Conclusions}

Overall, our review demonstrated that nutraceutical supplementation has lots of beneficial effects in patients with CCS, helping controlling risk factors in patients on optimal medical therapies who don't reach specific cut-off or are intolerant to some drug classes and acting directly on the ischemic myocardial tissue, through two mechanisms (cardiac energetics and ischemia-reperfusion damage) [82, 83]. We reviewed various high-quality studies but the knowledge about the effects of each molecule and the level of evidence for its use in patients with CCS is heterogeneous and further additional 
prospective studies regarding the effect of nutraceutical supplementation on ischemic myocardium are necessary before these strategies could be effectively used in clinical practice.

\section{Declarations}

Funding Open access funding provided by Università degli Studi di Milano - Bicocca within the CRUI-CARE Agreement. This work was supported by the A. De Gasperis Cardiology and Cardiac Surgery Foundation.

Conflict of interest All the authors declare that they have no conflict of interest.

\section{Ethical approval N/A.}

Informed consent N/A.

Open Access This article is licensed under a Creative Commons Attribution-NonCommercial 4.0 International License, which permits any non-commercial use, sharing, adaptation, distribution and reproduction in any medium or format, as long as you give appropriate credit to the original author(s) and the source, provide a link to the Creative Commons licence, and indicate if changes were made. The images or other third party material in this article are included in the article's Creative Commons licence, unless indicated otherwise in a credit line to the material. If material is not included in the article's Creative Commons licence and your intended use is not permitted by statutory regulation or exceeds the permitted use, you will need to obtain permission directly from the copyright holder. To view a copy of this licence, visit http://creativecommons.org/licenses/by-nc/4.0/.

\section{References}

1. Knuuti J, Wijns W, Saraste A, Capodanno D, Barbato E, FunckBrentano C, et al. 2019 ESC guidelines for the diagnosis and management of chronic coronary syndromes. Eur Heart J. 2020;41(3):407-77.

2. Kalra EK. Nutraceutical-definition and introduction. AAPS PharmSci. 2003;5(3):E25.

3. Poli A, Barbagallo CM, Cicero AFG, Corsini A, Manzato E, Trimarco B, et al. Nutraceuticals and functional foods for the control of plasma cholesterol levels. An intersociety position paper. Pharmacol Res. 2018;134:51-60.

4. Cicero AFG, Grassi D, Tocci G, Galletti F, Borghi C, Ferri C. Nutrients and nutraceuticals for the management of high normal blood pressure: an evidence-based consensus document. High blood Press Cardiovasc Prev. 2019;26(1):9-25.

5. Singh S, Schwarz K, Horowitz J, Frenneaux M. Cardiac energetic impairment in heart disease and the potential role of metabolic modulators: a review for clinicians. Circ Cardiovasc Genet. 2014;7(5):720-8.

6. Zhou L, Huang H, Yuan CL, Keung W, Lopaschuk GD, Stanley WC. Metabolic response to an acute jump in cardiac workload: effects on malonyl-CoA, mechanical efficiency, and fatty acid oxidation. Am J Physiol Heart Circ Physiol. 2008;294(2):H954-60.

7. Totzeck M, Hendgen-Cotta UB, Rassaf T. Nitrite-nitric oxide signaling and cardioprotection. Adv Exp Med Biol. 2017;982:335-46.

8. Ladilov YV, Siegmund B, Piper HM. Protection of reoxygenated cardiomyocytes against hypercontracture by inhibition of $\mathrm{Na}^{+} / \mathrm{H}^{+}$ exchange. Am J Physiol. 1995;268(4 Pt 2):H1531-9.
9. Chouchani ET, Pell VR, Gaude E, Aksentijević D, Sundier SY, Robb EL, et al. Ischaemic accumulation of succinate controls reperfusion injury through mitochondrial ROS. Nature. 2014;515(7527):431-5.

10. Rimm EB, Stampfer MJ, Ascherio A, Giovannucci E, Colditz GA, Willett WC. Vitamin E consumption and the risk of coronary heart disease in men. N Engl J Med. 1993;328(20):1450-6.

11. Riccioni G, Frigiola A, Pasquale S, Massimo DG, D’Orazio N. Vitamin $\mathrm{C}$ and $\mathrm{E}$ consumption and coronary heart disease in men. Front Biosci (Elite Ed). 2012;4:373-80.

12. Retsky KL, Freeman MW, Frei B. Ascorbic acid oxidation product(s) protect human low density lipoprotein against atherogenic modification. Anti-rather than prooxidant activity of vitamin $\mathrm{C}$ in the presence of transition metal ions. J Biol Chem. 1993;268(2):1304-9.

13. Thomas SR, Stocker R. Molecular action of vitamin E in lipoprotein oxidation: implications for atherosclerosis. Free Radic Biol Med. 2000;28(12):1795-805.

14. Babaev VR, Li L, Shah S, Fazio S, Linton MF, May JM. Combined vitamin $\mathrm{C}$ and vitamin $\mathrm{E}$ deficiency worsens early atherosclerosis in apolipoprotein E-deficient mice. Arterioscler Thromb Vasc Biol. 2010;30(9):1751-7.

15. Gunasekar P, Satish M, Dabestani P, Jiang W, Boosani C, Radwan $\mathrm{M}$, et al. Modulation of Cathepsin L expression in the coronary arteries of atherosclerotic swine. J Surg Res. 2019;243:460-8.

16. de Souza L, Junior A, Mancini Filho J, Pavan Torres R, Irigoyen $\mathrm{MC}$, Curi R. Pretreatment with fish oil attenuates heart ischaemia consequences in rats. Exp Physiol. 2017;102(11):1459-73.

17. da Silva GS, de Souza CW, da Silva L, Maciel G, Huguenin AB, de Carvalho M, et al. Effect of L-carnitine supplementation on reverse remodeling in patients with ischemic heart disease undergoing coronary artery bypass grafting: a randomized, placebocontrolled trial. Ann Nutr Metab. 2017;70(2):106-10.

18. Schönekess BO, Allard MF, Lopaschuk GD. Propionyl L-carnitine improvement of hypertrophied rat heart function is associated with an increase in cardiac efficiency. Eur J Pharmacol. 1995;286(2):155-66.

19. Wang Z-Y, Liu Y-Y, Liu G-H, Lu H-B, Mao C-Y. l-Carnitine and heart disease. Life Sci. 2018;194:88-97.

20. Scarabelli TM, Townsend PA, Chen Scarabelli C, Yuan Z, McCauley RB, Di Rezze J, et al. Amino acid supplementation differentially modulates STAT1 and STAT3 activation in the myocardium exposed to ischemia/reperfusion injury. Am J Cardiol. 2008;101(11A):63E-68E.

21. Kulthinee S, Wyss JM, Roysommuti S. Taurine supplementation prevents the adverse effect of high sugar intake on arterial pressure control after cardiac ischemia/reperfusion in female rats. Adv Exp Med Biol. 2015;803:597-611.

22. Pranav Nayak B, Ganesha KR, Minaz N, Razdan R, Goswami SK. Phloroglucinol, a nutraceutical for IR-induced cardiac damage in diabetic rats. Anim Model Exp Med. 2019;2(3):210-6.

23. Sabe AA, Sadek AA, Elmadhun NY, Dalal RS, Robich MP, Bianchi $\mathrm{C}$, et al. Investigating the effects of resveratrol on chronically ischemic myocardium in a swine model of metabolic syndrome: a proteomics analysis. J Med Food. 2015;18(1):60-6.

24. Quintieri AM, Baldino N, Filice E, Seta L, Vitetti A, Tota B, et al. Malvidin, a red wine polyphenol, modulates mammalian myocardial and coronary performance and protects the heart against ischemia/reperfusion injury. J Nutr Biochem. 2013;24(7):1221-31.

25. Rivera K, Salas-Pérez F, Echeverría G, Urquiaga I, Dicenta S, Pérez D, et al. Red wine grape pomace attenuates atherosclerosis and myocardial damage and increases survival in association with improved plasma antioxidant activity in a murine model of lethal ischemic heart disease. Nutrients. 2019;11(9). 
26. Shecterle LM, Terry KR, St Cyr JA. The patented uses of D-ribose in cardiovascular diseases. Recent Pat Cardiovasc Drug Discov. 2010;5(2):138-42.

27. Gonzalez GE, Rabald S, Briest W, Gelpi RJ, Seropian I, Zimmer $\mathrm{H}-\mathrm{G}$, et al. Ribose treatment reduced the infarct size and improved heart function after myocardial infarction in rats. Cell Physiol Biochem Int J Exp Cell Physiol Biochem Pharmacol. 2009;24(3-4):211-8.

28. Kurutas EB. The importance of antioxidants which play the role in cellular response against oxidative/nitrosative stress: current state. Nutr J. 2016;15(1):71.

29. Salehi B, Berkay Yılmaz Y, Antika G, Boyunegmez Tumer T, Fawzi Mahomoodally M, Lobine D, et al. Insights on the use of $\alpha$-lipoic acid for therapeutic purposes. Biomolecules. 2019;9(8).

30. Ghibu S, Richard C, Vergely C, Zeller M, Cottin Y, Rochette L. Antioxidant properties of an endogenous thiol: Alpha-lipoic acid, useful in the prevention of cardiovascular diseases. J Cardiovasc Pharmacol. 2009;54(5):391-8.

31. Van Hoose PM, Kelm NQ, Piell KM, Cole MP. Conjugated linoleic acid and nitrite attenuate mitochondrial dysfunction during myocardial ischemia. J Nutr Biochem. 2016;34:8-16.

32. Ueshima K. Magnesium and ischemic heart disease: a review of epidemiological, experimental, and clinical evidences. Magn Res. 2005;18(4):275-84

33. Kutil B, Ostadal P, Vejvoda J, Kukacka J, Cepova J, Alan D, et al. Alterations in serum selenium levels and their relation to troponin I in acute myocardial infarction. Mol Cell Biochem. 2010;345(1-2):23-7.

34. Benstoem C, Goetzenich A, Kraemer S, Borosch S, Manzanares W, Hardy G, et al. Selenium and its supplementation in cardiovascular disease-what do we know? Nutrients. 2015;7(5):3094-118.

35. Choi S, Liu X, Pan Z. Zinc deficiency and cellular oxidative stress: prognostic implications in cardiovascular diseases. Acta Pharmacol Sin. 2018;39(7):1120-32.

36. Lal A. Effect of zinc sulphate on infarct size in experimental myocardial infarction in dogs. Indian J Med Res. 1991;94:316-9.

37. Karagulova G, Yue Y, Moreyra A, Boutjdir M, Korichneva I. Protective role of intracellular zinc in myocardial ischemia/reperfusion is associated with preservation of protein kinase $\mathrm{C}$ isoforms. J Pharmacol Exp Ther. 2007;321(2):517-25.

38. Xu Z, Kim S, Huh J. Zinc plays a critical role in the cardioprotective effect of postconditioning by enhancing the activation of the RISK pathway in rat hearts. J Mol Cell Cardiol. 2014;66:12-7.

39. Bronzato S, Durante A. Dietary supplements and cardiovascular diseases. Int J Prev Med. 2018;9:80.

40. Wang YZ, Deng L, Xu HC, Zhang Y, Liang ZQ. Laparoscopy versus laparotomy for the management of early stage cervical cancer. BMC Cancer. 2015. https://doi.org/10.1186/s12885-015-1818-4.

41. Raygan F, Taghizadeh M, Mirhosseini N, Akbari E, Bahmani F, Memarzadeh MR, et al. A comparison between the effects of flaxseed oil and fish oil supplementation on cardiovascular health in type 2 diabetic patients with coronary heart disease: a randomized, double-blinded, placebo-controlled trial. Phytother Res. 2019;33(7):1943-51.

42. Gabrielová E, Bartošíková L, Nečas J, Modrianský M. Cardioprotective effect of 2,3-dehydrosilybin preconditioning in isolated rat heart. Fitoterapia. 2019;132:12-21.

43. Szucs G, Bester DJ, Kupai K, Csont T, Csonka C, Esterhuyse AJ, et al. Dietary red palm oil supplementation decreases infarct size in cholesterol fed rats. Lipids Health Dis. 2011;10:103.

44. Salloum FN, Sturz GR, Yin C, Rehman S, Hoke NN, Kukreja $\mathrm{RC}$, et al. Beetroot juice reduces infarct size and improves cardiac function following ischemia-reperfusion injury: possible involvement of endogenous H2S. Exp Biol Med (Maywood). 2015;240(5):669-81.
45. Bhatt DL, Steg PG, Miller M, Brinton EA, Jacobson TA, Ketchum $\mathrm{SB}$, et al. Effects of icosapent ethyl on total ischemic events: from REDUCE-IT. J Am Coll Cardiol. 2019;73(22):2791-802.

46. Morrison D, Hughes J, Della Gatta PA, Mason S, Lamon S, Russell AP, et al. Vitamin C and E supplementation prevents some of the cellular adaptations to endurance-training in humans. Free Radic Biol Med. 2015;89:852-62.

47. Albert CM, Campos H, Stampfer MJ, Ridker PM, Manson JE, Willett WC, et al. Blood levels of long-chain n-3 fatty acids and the risk of sudden death. N Engl J Med. 2002;346(15):1113-8.

48. Ostadmohammadi V, Milajerdi A, Ghayour-Mobarhan M, Ferns G, Taghizadeh M, Badehnoosh B, et al. The effects of vitamin D supplementation on glycemic control, lipid profiles and C-reactive protein among patients with cardiovascular disease: a systematic review and meta-analysis of randomized controlled trials. Curr Pharm Des. 2019;25(2):201-10.

49. Albert CM, Hennekens CH, O'Donnell CJ, Ajani UA, Carey VJ, Willett WC, et al. Fish consumption and risk of sudden cardiac death. JAMA. 1998;279(1):23-8.

50. Bazzano LA, Reynolds K, Holder KN, He J. Effect of folic acid supplementation on risk of cardiovascular diseases: a meta-analysis of randomized controlled trials. JAMA. 2006;296(22):2720-6.

51. Lemaitre RN, King IB, Mozaffarian D, Kuller LH, Tracy RP, Siscovick DS. n-3 Polyunsaturated fatty acids, fatal ischemic heart disease, and nonfatal myocardial infarction in older adults: the Cardiovascular Health Study. Am J Clin Nutr. 2003;77(2):319-25.

52. Burr ML, Fehily AM, Gilbert JF, Rogers S, Holliday RM, Sweetnam PM, et al. Effects of changes in fat, fish, and fibre intakes on death and myocardial reinfarction: diet and reinfarction trial (DART). Lancet (London, England). 1989;2(8666):757-61.

53. Goodfellow J, Bellamy MF, Ramsey MW, Jones CJ, Lewis MJ. Dietary supplementation with marine omega-3 fatty acids improve systemic large artery endothelial function in subjects with hypercholesterolemia. J Am Coll Cardiol. 2000;35(2):265-70.

54. Calder PC. Omega 3 polyunsaturated fatty acids, inflammation and immunity. World Rev Nutr Diet. 2001;88:109-16.

55. Popoff F, Balaciano G, Bardach A, Comandé D, Irazola V, Catalano HN, et al. Omega 3 fatty acid supplementation after myocardial infarction: a systematic review and meta-analysis. BMC Cardiovasc Disord. 2019;19(1):136.

56. Bhatt DL. REDUCE-IT. Eur Heart J. 2019;40(15):1174-5.

57. Ganda OP, Bhatt DL, Mason RP, Miller M, Boden WE. Unmet need for adjunctive dyslipidemia therapy in hypertriglyceridemia management. J Am Coll Cardiol. 2018;72(3):330-43.

58. Ference BA, Kastelein JJP, Ray KK, Ginsberg HN, Chapman MJ, Packard CJ, et al. Association of triglyceride-lowering LPL variants and LDL-C-lowering LDLR variants with risk of coronary heart disease. JAMA. 2019;321(4):364-73.

59. Rizos I. Three-year survival of patients with heart failure caused by dilated cardiomyopathy and L-carnitine administration. Am Heart J. 2000;139(2 Pt 3):S120-3.

60. Hoseini A, Namazi G, Farrokhian A, Reiner Ž, Aghadavod E, Bahmani F, et al. The effects of resveratrol on metabolic status in patients with type 2 diabetes mellitus and coronary heart disease. Food Funct. 2019;10(9):6042-51.

61. Jiang W, Wei H, He B. Dietary flavonoids intake and the risk of coronary heart disease: a dose-response meta-analysis of 15 prospective studies. Thromb Res. 2015;135(3):459-63.

62. Arevström L, Bergh C, Landberg R, Wu H, Rodriguez-Mateos A, Waldenborg M, et al. Freeze-dried bilberry (Vaccinium myrtillus) dietary supplement improves walking distance and lipids after myocardial infarction: an open-label randomized clinical trial. Nutr Res. 2019;62:13-22.

63. Pliml W, von Arnim T, Stäblein A, Hofmann H, Zimmer HG, Erdmann E. Effects of ribose on exercise-induced ischaemia 
in stable coronary artery disease. Lancet (London, England). 1992;340(8818):507-10.

64. Omran H, Illien S, MacCarter D, St Cyr J, Lüderitz B. D-Ribose improves diastolic function and quality of life in congestive heart failure patients: a prospective feasibility study. Eur J Heart Fail. 2003;5(5):615-9.

65. Rodríguez-Moran M, Guerrero-Romero F. Oral magnesium supplementation improves the metabolic profile of metabolically obese, normal-weight individuals: a randomized double-blind placebo-controlled trial. Arch Med Res. 2014;45(5):388-93.

66. Shechter M, Merz CN, Paul-Labrador M, Meisel SR, Rude RK, Molloy MD, et al. Oral magnesium supplementation inhibits platelet-dependent thrombosis in patients with coronary artery disease. Am J Cardiol. 1999;84(2):152-6.

67. Teo KK, Yusuf S. Role of magnesium in reducing mortality in acute myocardial infarction, a review of the evidence. Drugs. 1993;46(3):347-59.

68. Steinbrenner H, Bilgic E, Pinto A, Engels M, Wollschläger L, Döhrn L, et al. Selenium pretreatment for mitigation of ischemia/ reperfusion injury in cardiovascular surgery: influence on acute organ damage and inflammatory response. Inflammation. 2016;39(4):1363-76.

69. Gharipour M, Sadeghi M, Behmanesh M, Salehi M, Roohafza $\mathrm{H}$, Nezafati $\mathrm{P}$, et al. Proposal of a study protocol of a preliminary double-blind randomized controlled trial. Verifying effects of selenium supplementation on selenoprotein $p$ and s genes expression in protein and mRNA levels in subjects with coronary artery disease: selenegen. Acta Biomed. 2019;90(1):44-50.

70. Yang YJ, Choi BY, Chun B-Y, Kweon S-S, Lee Y-H, Park PS, et al. Dietary zinc intake is inversely related to subclinical atherosclerosis measured by carotid intima-media thickness. Br J Nutr. 2010;104(8):1202-11.

71. Singh RB, Niaz MA, Rastogi SS, Bajaj S, Gaoli Z, Shoumin $Z$. Current zinc intake and risk of diabetes and coronary artery disease and factors associated with insulin resistance in rural and urban populations of North India. J Am Coll Nutr. 1998;17(6):564-70.

72. Schwingshackl L, Boeing H, Stelmach-Mardas M, Gottschald M, Dietrich S, Hoffmann G, et al. Dietary supplements and risk of cause-specific death, cardiovascular disease, and cancer: a systematic review and meta-analysis of primary prevention trials. Adv Nutr. 2017;8(1):27-39.
73. Low WI, Ikram H. Plasma zinc in acute myocardial infarction Diagnostic and prognostic implications. Br Heart J. 1976;38(12):1339-42.

74. Liu B, Cai Z-Q, Zhou Y-M. Deficient zinc levels and myocardial infarction : association between deficient zinc levels and myocardial infarction: a meta-analysis. Biol Trace Elem Res. 2015;165(1):41-50.

75. Huang L, Teng T, Zhao J, Bian B, Yao W, Yu X, et al. The relationship between serum zinc levels, cardiac markers and the risk of acute myocardial infarction by zinc quartiles. Heart Lung Circ. 2018;27(1):66-72.

76. Alehagen U, Aaseth J, Johansson P. Reduced cardiovascular mortality 10 years after supplementation with selenium and Coenzyme Q10 for 4 years: follow-up results of a prospective randomized double-blind placebo-controlled trial in elderly citizens. PLoS ONE. 2015;10(12):e0141641.

77. Rivellese AA, Ciciola P, Costabile G, Vetrani C, Vitale M. The possible role of nutraceuticals in the prevention of cardiovascular disease. High Blood Press Cardiovasc Prev. 2019;26(2):101-11.

78. Martelli A, Testai L, Colletti A, Cicero AFG. Coenzyme Q(10): clinical applications in cardiovascular diseases. Antioxidants (Basel, Switzerland). 2020;9(4).

79. Qu H, Meng Y-Y, Chai H, Liang F, Zhang J-Y, Gao Z-Y, et al. The effect of statin treatment on circulating coenzyme Q10 concentrations: an updated meta-analysis of randomized controlled trials. Eur J Med Res. 2018;23(1):57.

80. Cicero AFG, Colletti A, Fogacci F, Bove M, Rosticci M, Borghi C. Effects of a combined nutraceutical on lipid pattern, glucose metabolism and inflammatory parameters in moderately hypercholesterolemic subjects: a double-blind, cross-over, randomized clinical trial. High Blood Press Cardiovasc Prev. 2017;24(1):13-8.

81. Caso G, Kelly P, McNurlan MA, Lawson WE. Effect of coenzyme q10 on myopathic symptoms in patients treated with statins. Am J Cardiol. 2007;99(10):1409-12.

82. Volpe R, Sotis G. Nutraceuticals: definition and epidemiological rationale for their use in clinical practice. High Blood Press Cardiovasc Prev. 2015;22(3):199-201.

83. Cicero AFG, Colletti A, von Haehling S, Vinereanu D, BieleckaDabrowa A, Sahebkar A, et al. Nutraceutical support in heart failure: a position paper of the International Lipid Expert Panel (ILEP). Nutr Res Rev. 2020;1-25. 